\title{
Instrucción de estrategias de lectura digital mediante modelado por video
}

\section{Instruction of digital reading strategies by means of video modeling}

Fecha de recepción: 23/11/2014

Fecha de aceptación: 02/12/2015

\author{
Ladislao Salmerón $^{1}$, Ana Llorens ${ }^{2}$ e Inmaculada Fajardo ${ }^{1}$ \\ ${ }^{1}$ Universitat de València \\ ${ }^{2}$ Florida Universitaria y Universidad Internacional de la Rioja (UNIR)
}

\section{resumen/abstract:}

Los resultados de las pruebas de evaluación internacionales indican que las habilidades de lectura digital de nuestros adolescentes están por debajo de la media de los países de la OCDE. Este es el punto de partida del presente estudio, que analiza la efectividad de un programa de instrucción basado en la técnica del modelado por video a partir de movimientos oculares o EMMEs para la enseñanza de las estrategias de lectura en entornos digitales a un grupo de estudiantes de secundaria. Los resultados muestran un aumento en la frecuencia de estrategias de lectura digital mencionadas por los estudiantes después de la intervención, enespecial,planificación de la búsqueda y la lectura y revisión delplan de acción cuando esnecesario. En el artículo se discute como EMME podría ser una técnica efectiva para la instrucción de tareas cognitivas complejas en el contexto escolar que además permitiera la aplicación individualizada y la reducción de la carga del docente.

Results from international assessment tests show that the skills for digital reading of our adolescents are below the average of OECD countries. This is the starting point of our study that analyzes the effectiveness of an instructional program based on the eye-movements video modelling technique (EMMEs) to teach reading strategies in digital contexts, to a group of secondary education students. Results show an increment in the frequency of strategies mentioned after the intervention, in particular, planning the search and the reading andrevising the action plan when necessary. Finally, we discuss how EMMEs could be an effective technique for the instruction of complex cognitive tasks in the academic context, which may allow an individualized application and a reduction on the teachers' load.

\section{palabras clave/keywords:}

estrategias lectura digital, video modelado de movimientos oculares, educación secundaria

digital reading strategies, eye-movement modelling examples, secondary education

Agradecimientos:

Esta investigación ha sido financiada por un proyecto de investigación de la Secretaría General de Universidades (EDU2014-59422). Agradecemos a los alumnos y al resto de la comunidad educativa su participación en el estudio.

\section{Introducción}

Si algo caracteriza a los denominados millenials, o adolescentes actuales, es su rápida transición del mundo analógico, predominante en generaciones anteriores, al mundo digital. Esta transición se ha dado en multitud de esferas de la vida cotidiana, y es particularmente marcada en el ámbito de la lectura. En una encuesta reciente sobre hábitos lectores de los españoles, un 19,6\% de los lectores entre 14-24 años declaró que sólo leía libros, revistas y prensa en dispositivos digitales, frente a un 9,3\% que sólo lo hacía en papel (CONECTA, 
2014). Pese a todo, los resultados de las pruebas de evaluación internacionales indican que las habilidades de lectura digital de nuestros adolescentes están por debajo de la de los estudiantes de los países de la OCDE (OCDE, 2013).Quizá por ello sorprenda que en el contexto escolar la instrucción en comprensión lectora está aún centrada en los textos en papel. Por tanto, en el contexto actual resulta necesario explorar diversas técnicas instruccionales para la enseñanza de las estrategias de lectura en entornos digitales. En este estudio se presenta y evalúa una técnica innovadora para la instrucción de estrategias de lectura digital, concretamente el video modelado. Para ello, en primer lugar se analiza la literatura sobre la enseñanza de la lectura digital. A continuación se introducen los estudios previos sobre video modelado, y finalmente se presenta una experiencia piloto realizada con el objetivo de testar la eficacia de la técnica de video modelado para el aprendizaje de estrategias de lectura digital en adolescentes.

\section{Instrucción de estrategias de lectura digital}

Hasta qué punto la lectura digital requiere una instrucción diferenciada de la tradicional comprensión de textos en papel constituye un objeto de debate en la actualidad. En un extremo del debate, los partidarios de la corriente de las Nuevas Alfabetizaciones (Coiro, 2011), consideran que es necesario instruir de forma radicalmente distinta la lectura digital, porque ésta requiere habilidades que no están presentes, o al menos en menor medida, en la lectura en papel. La lectura digital exige que el lector tenga un plan de lectura inicial claro que debe evaluarse constantemente para evitar perderse en el ciberespacio, sea capaz de seleccionar páginas web relevantes para su plan y de abandonar rápidamente páginas abiertas que finalmente resulten irrelevantes, y evalúe de forma crítica la veracidad de las páginas y de la información.(Brand-Gruwel, Wopereis y Walraven, 2009). Estas habilidades son especialmente relevantes en la lectura digital por las particularidades de Internet, donde existen multitudes de páginas web interconectadas para una misma temática, con niveles muy diversos de calidad, debido a que cualquiera puede escribir y publicar en la red. En un punto intermedio del debate, los partidarios del modelo de mediación, proponen que aunque la lectura digital tiene sus particularidades, comparte muchas habilidades con la lectura en papel, por lo que su instrucción debe ser complementaria (Salmerón y García, 2011). En esta línea, diversos estudios tanto a pequeña como a gran escala indican que los adolescentes con buenas habilidades de comprensión lectora en papel navegan de manera más eficiente en textos digitales (por ejemplo, acceden a más páginas relacionadas con su plan de lectura), lo que conlleva que obtengan una mejor comprensión en tareas de lectura digital. En este debate, no conocemos posturas en el extremo opuesto que aboguen por no incluir una instrucción en estrategias de lectura digital, y que apuesten únicamente por la enseñanza de la comprensión lectora en papel.

Por tanto, pensamos que es necesario incorporar en nuestro sistema educativo una enseñanza de la lectura digital que complemente a los programas de lectura en papel, sin llegar a reemplazarlos. Durante la pasada década se han propuesto y contrastado diversos métodos de enseñanza de la lectura digital en adolescentes(Argelagós y Pifarré, 2012; De Vries, van der Meij y Lazonder, 2008; Kuiper, Volman, y Terwel, 2008, 2009; Kuo y Hwang, 2008; 
Walraven, Brand-Gruwel, y Boshuizen, 2010). En general, se trata de programas de larga duración y en su mayor parte integradas en el currículum escolar, donde por lo general juega un papel relevante la enseñanza explícita de estrategias de lectura digital.

Aunque los programas han demostrado cierta utilidad para la instrucción de estrategias de lectura digital, éstos incluyen diversas técnicas instruccionales, por lo que no resulta sencillo determinar la efectividad de cada una de ellas. De Vries et al. (2008) utilizaron plantillas de apoyo para guiar a un grupo de estudiantes de $5^{\circ}$ y $6^{\circ}$ de educación primaria (EP), que trabajan en parejas. En las plantillas los estudiantes debían escribir todo lo que recordaran de la pregunta planteada por el profesor, con el objetivo de activar sus conocimientos previos. Kuiper et al. $(2008,2009)$ llevaron a cabo un programa de dos meses integrado en el currículum escolar, en varios centros de $5^{\circ}$ de EP. Las profesoras explicaban qué estrategia se trabajaba en cada sesión, y los alumnos lo ponían en práctica en parejas. Por su parte, Kuoy Hwang(2008) trabajaron con estudiantes de $5^{\circ}$ de EP durante 5 semanas. Su programa, basado en el principiode andamiaje, pretendía que los alumnos activaran sus conocimientos durante la lectura, abstrajeran las técnicas utilizadas, y reflexionaran sobre las mismas. El andamiaje se daba en las primeras fases, y se retiraba a partir de la tercera sesión.

Otros programas han trabajado las estrategias de lectura digital con estudiantes de educación secundaria (ESO). Argelagós y Pifarré (2012) llevaron a cabo una intervención de dos años con estudiantes de $1^{\circ}$ y $2^{\circ}$ de ESO. El programa instruccional tenía una orientación socio-constructivista, y estaba integrado en el currículum del centro. Los estudiantes realizaban tareas estructuradas con unas guías o andamios para fomentar los pasos de activación de conocimientos, guía durante la tarea y reflexión final (WebQuest). Por su parte, Walraven et al. (2008) evaluaron la efectividad de dos tipos de programas instruccionales con estudiantes de 14-15 años, en un programa de 15 sesiones. Uno de los grupos utilizaba una plantilla con una descripción paso a paso de las estrategias necesarias para resolver las tareas en Internet. Otro de los grupos utilizó como apoyo instruccional un mapa conceptual que representaba un árbol de decisión para ayudar a los estudiantes a decidir qué paso seguir en cada momento.

Nuestra revisión indica que los programas actuales utilizan una gran diversidad de técnicas instruccionales para la enseñanza de estrategias de lectura digital. Ninguno de ellos, sin embargo, utiliza el modelado por video.

\section{Modelado por video para la enseñanza}

Los adolescentes utilizan con frecuencia videos instruccionales en sitios web como YouTube para el aprendizaje informal, como por ejemplo aprender a combinar bien la ropa, o aprender a solucionar una situación problemática en un juego en línea. Los videos instruccionales también tienen su importancia en la enseñanza formal, incluyendo clases magistrales, videos de casos o tutoriales (Spires, Hervey, Morris y Stelpflug, 2012). Una de las mayores ventajas de los videos instruccionales es que se pueden consultar en cualquier momento, y se pueden ver en repetidas ocasiones sin que el tutor / modelo se canse. Los videos, particularmente los tutoriales, permiten modelar la conducta estratégica de un experto, lo que los convierte en una técnica adecuada para la instrucción de estrategias. Desde 
la teoría social del aprendizaje (Bandura, 1986) se ha constatado la eficacia de los videos en los que un experto modela, mediante la explicación y puesta en práctica, los procedimientos necesarios para la realización de una tarea compleja. El aprendizaje por modelado se ha probado en numerosas áreas, como las matemáticas, lectura o escritura (para una revisión reciente ver Van Gogh y Rummel, 2010).

Este tipo de aprendizaje no implica meramente visionar un video. Bandura (1986) concluye que para que el aprendizaje por modelado sea efectivo se deben dar cuatro condicionantes en relación al aprendiz: 1) debe prestar atención a la conducta relevante del modelo, 2) debe elaborar la información en su memoria, 3) debe ser capaz de reproducir la conducta modelada, y 4) debe estar motivado para poner en práctica el aprendizaje modelado. Estas precondiciones se deben tener en cuenta en el diseño instruccional de cualquier programa que incluya el modelado por video.Por ejemplo, en nuestro caso de estudio, las estrategias de lectura, el modelo verbaliza los pasos mentales necesarios realizar la estrategia, con el objetivo de que el estudiante preste atención a la conducta relevante que se quiere modela. Sin embargo, no siempre resulta sencillo verbalizar todos los pasos mentales implicados en la utilización experta de estrategias cognitivas complejas. Por ejemplo, mientras un lector avanzado piensa "Voy a explorar rápidamente las secciones de la página web para decidir si ésta página contiene información relevante para mi objetivo de estudio", a la vez está moviendo sus ojos a lo largo de la página, por ejemplo, leyendo rápidamente los enunciados, hiperenlaces e imágenes presente.

La técnica del modelado de ejemplos a partir de movimientos oculares (EMME, por sus siglas en inglés) permite modelar conjuntamente en un video los pensamientos que guían una conducta estratégica, así como la inspección visual que hace el experto durante la aplicación de dicha estrategia. Esta técnica instruccional ha sido propuesta recientemente por la investigadora HalszkaJarodzka y ha sido aplicada con éxito a la enseñanzade temáticas como el diagnóstico visual en bebés (Jarodzka et al., 2012), la locomoción de los peces (Jarodzka, van Gog, Dorr, Scheiter y Gerjets, 2013), la resolución de problemas visuales (Van Gog, Jarodzka, Scheiter, Gerjets y Paas, 2009), o la integración texto con imágenes (Mason, Pluchino y Tornatora $(2015 a, b)$.

En estos estudios, los EMME fueron utilizados para guiar la atención de los estudiantes en tareas visualmente complejas, pero que requerían estrategias relativamente sencillas (por ejemplo, "Observa el tipo de movimiento de las aletas del pez para clasificarlo"). En el presente trabajo, queremos poner a prueba la efectividad de los EMME para la instrucción de estrategias cognitivas complejas, las estrategias de navegación en la lectura digital.

\section{Objetivos del Estudio}

El presente estudio examina la efectividad de un breve entrenamiento para fomentar estrategias de lectura digital en documentos digitales similares a las páginas que incluye Wikipedia. El entrenamientose divide en tres fases: modelado, práctica y reflexión (Coiro, 2011), que se detallan en apartados posteriores del artículo. La característica más novedosa del procedimiento de instrucción planteado es el usodelos EMME. Esta técnica se incluye como parte de una tarea de contraste de casos dirigida a mejorar tanto el aprendizaje como 
la transferencia de las estrategias de lectura digital (e.g., Salmerón, 2013). Como punto de partida, se plantea que el entrenamiento basado en EMMEs y contraste de casos resultará efectivo para incrementar el conocimiento de los estudiantes sobre estrategias de búsqueda de información y su posible aplicación en situaciones de lectura digital en Educación Secundaria.

\section{Método}

\section{Participantes}

Los estudiantes que participaron en el presente estudio pertenecían a un centro público de la provincia de Valencia. Previamente a la aplicación del estudio se requirió un consentimiento informado a las familias de cada uno de los alumnos. La muestra definitiva se compuso de 20 estudiantes de diferentes cursos de toda la etapa de Educación Secundaria Obligatoria (edad media $=13.5, D T=1.32)$. El 65\% de los estudiantes eran chicas, y todos ellos utilizaban el castellano como lengua materna.

\section{Entrenamiento en Estrategias de Lectura Digital}

El entrenamiento se diseñó para ser aplicado en aproximadamente una hora, siguiendo un procedimiento dividido en diferentes pasos o fases. En concreto, se pueden distinguir tres fases principales: modelado, práctica y reflexión(Coiro, 2011).La fase de modelado se iniciaba con una breve instrucción explícita sobre sobre las características de la lectura en documentos digitales, y la aplicación de estrategias necesarias para seleccionar y procesar la información relevante para la tarea de aprendizaje. En concreto, para explicar las estrategias de lectura la presentación se dividió en tres secciones que correspondían a las tres estrategias principales propuestas por Coiro y Dobler (2007) para leer, buscar y localizar información en documentos digitales: planificar (establecer una meta y desarrollar un plan mental);evaluar la búsqueda de información (anticipar qué información contiene una sección o enlace del documento, y adaptar la lectura según la relevancia de la información, explorando superficialmente el texto o dedicando más tiempo a comprender en profundidad la información); y revisar (supervisar y valorar la relevancia de una elección o respuesta). Por último, se incluyó información sobre la importancia de utilizar los conocimientos previos sobre el tema del documento digital o la estructura del mismo para buscar información de forma efectiva. Además, cada uno de los conocimientos declarativos se acompañaba de un ejemplo con el objetivo de ilustrar y modelar la correcta aplicación las estrategias de búsqueda.

La instrucción explícita se conducía y explicaba por uno de los investigadores que seguía un estricto guion de presentación y resolvía las dudas de los estudiantes cuando lo requerían. Una vez los estudiantes se habían familiarizado con los conceptos clave del entrenamiento y conocían la forma en la que se deben aplicar las estrategias de lectura digital, se presentaban dos ejemplos usando vídeos elaborados mediante la técnica de los EMME. Cada uno de los vídeos mostraba la ejecución de un estudiante que leía información en un texto de Wikipedia para responder una pregunta. Los vídeos mostraban el texto del documento y un punto que indicaba la posición de los ojos de los estudiantes, es decir, indicabaqué estaba 
leyendo el alumno en cada momento. Además, se acompañaba con una narración en la que los estudiantes del ejemplo manifestaban los pasos y decisiones que iban tomando durante la búsqueda de información. De esa forma se introducía la siguiente tarea que debían realizar en la fase de práctica.

La fase de práctica incluía 6 tareas de contraste de casos breves similares a los casos del ejemplo anterior. En cada tarea se presentaban dos EMME. En todas ellas, uno de los casos aplicaba las estrategias de lectura correctamente (junto con alguna estrategia incorrecta), mientas que en el otro el estudiante cometía varios errores durante la lectura de información (junto con alguna estrategia correcta).

La tarea de los estudiantes de Secundaria era discutir por parejas, intentando llegar a un acuerdo sobre: a) cuál de los dos estudiantes delos EMME tenía más posibilidades de contestar correctamente una pregunta de comprensión sobre el contenido del documento, y b) justificar su respuesta examinando si se habían aplicado correctamente las estrategias de planificar, evaluar la búsqueda de información y revisar, y por qué consideraban que se habían aplicado o no cada una de ellas en ambos ejemplos. El audio de las discusiones de todas las parejas de estudiantes fue grabado para su posterior análisis.

Finalmente, en la fase de reflexión los estudiantes recibían una retroalimentación formativa tras discutir sobre cada uno de los 6 contrastes de casos. Esta retroalimentación informaba a los estudiantes sobre las estrategias correctas o incorrectas mostradas en cada uno de los dos vídeos de modelado de ejemplos. Además, se incitaba a los estudiantes a revisar cuidadosamente si sus conclusiones durante la discusión por parejas coincidían con la resolución del caso que facilitaba la retroalimentación (Nicol y Macfarlane-Dick, 2006).

\section{Medidas}

Cuestionario declarativo sobre estrategias de búsqueda (pre-post test). Para evaluar el conocimiento de los estudiantes sobre las diferentes estrategias de navegación en documentos digitales se elaboró un cuestionario, basado en Eagleton y Dobler (2012), que demandaba elaborar un listado de estrategias necesaria para la búsqueda de información en Wikipedia. En concreto, se planteaba la siguiente situación: "Imagina que alguien debe responder una pregunta sobre el contenido de un texto de Wikipedia". A continuación, se pedía a los estudiantes que enumeraran cada uno de los pasos que una persona necesita poner en marcha para buscar información en un documento de Wikipedia. Este cuestionario se aplicó antes y después del entrenamiento en estrategias de lectura digital.

Prueba de Eficiencia Lectora. La Prueba de Eficiencia Lectora (PEL) (Carrillo y Marín, 1997) permite la evaluación rápida del nivel de lectura de los estudiantes. Se compone de 64 frases incompleta que los alumnos deben completar eligiendo entre cuatro palabras posibles.

Calidad de las discusiones en la tarea de contrastar casos. Para analizar la calidad de las discusiones durante el contraste de casos se analizaron y categorizaron las verbalizaciones de cada una de las parejas de estudiantes. En concreto, se establecieron una serie de categorías atendiendo al contenido de la discusión (nivel de justificación de las ideas) y al tipo de interacción entre los estudiantes (grado de intercambio de ideas) (Spatariu, Hartley, y 
Bendixen, 2004). Las categorías y puntuaciones resultantes de la combinación de ambas dimensiones se incluyen en la Tabla 1.

Tabla 1. Categorías para el análisis de las discusiones según contenido e interacción entre los estudiantes

\begin{tabular}{|lc|}
\hline Categoría & Puntuación \\
\hline $\begin{array}{l}\text { Discusión justificada. Los estudiantes intercambian ideas sobre } \\
\text { las estrategias mostradas en los vídeos de ejemplo y justifican su } \\
\text { respuesta con información sobre estrategias de búsqueda digital }\end{array}$ & 6 \\
correctas. & 5 \\
\hline $\begin{array}{l}\text { Discusión sin justificación completa. Los estudiantes intercam- } \\
\text { bian ideas sobre las estrategias mostradas en los vídeos de ejem- } \\
\text { plo pero solo justifican correctamente algunas de sus observacio- }\end{array}$ & \\
nes. & 4 \\
\hline $\begin{array}{l}\text { Discusión Sí/No. Los estudiantes intercambian ideas sobre las } \\
\text { estrategias mostradas en los vídeos de ejemplo pero solamente } \\
\text { comentan si una determinada estrategia aparece o no. }\end{array}$ & \\
\hline $\begin{array}{l}\text { Discusión por turnos. Los estudiantes explican sus ideas sobre } \\
\text { las estrategias de búsqueda por turnos pero no intercambian sus } \\
\text { observaciones. }\end{array}$ & 3 \\
\hline $\begin{array}{l}\text { Un estudiante realiza la tarea. Solamente un estudiante manifies- } \\
\text { ta sus observaciones uno de los estudiantes justificando correc- } \\
\text { tamente. }\end{array}$ & 2 \\
\hline $\begin{array}{l}\text { Un estudiante realiza la tarea sin justificar. Solamente un estu- } \\
\text { diante manifiesta sus observaciones pero no justifica su punto de } \\
\text { vista. }\end{array}$ & 1 \\
\hline Los estudiantes no realizan la tarea correctamente. & 0 \\
\hline
\end{tabular}

\section{Procedimiento}

La aplicación completa del estudio exploratorio duró aproximadamente una hora y media. En primer lugar, los estudiantes completaron individualmente la Prueba de Eficiencia Lectora y a continuación, el cuestionario declarativo sobre estrategias de búsqueda. Entonces, se inició el entrenamiento en el orden descrito anteriormente: instrucción explícita con ejemplos de modelado y realización práctica de 6 tareas contraste de EMMEs.Por último, los estudiantes completaron de nuevo el cuestionario declarativo sobre estrategias de lectura digital. 


\section{Diseño}

Se empleó un diseño intra-sujetos en el que todos los estudiantes recibían el entrenamiento completo en estrategias de lectura digital. Las variables dependientes analizadas incluyen los resultados de un cuestionario de evaluación del tipo de estrategias empleadas para contestar preguntas a partir de información de un documento similar a Wikipedia, aplicado antes y después del entrenamiento. Además, se analizaron las verbalizaciones de las parejas de estudiantes durante el contraste de casos de estrategias presentadas en los EMME.

\section{Resultados}

\section{Aprendizaje de Conocimiento Declarativo sobre Estrategias de Lectura Digital}

En primer lugar analizamos el aprendizaje de conocimiento declarativo sobre estrategias de lectura digital, comparando las respuestas de los alumnos antes y después de la intervención (ver tabla 2). Específicamente, cada una de sus respuestas se categorizó como como 'técnicas generales' (aspectos técnicos "encender el ordenador o abrir el buscador de Internet" y/o a aspectos de la tarea de contestación de preguntas "leer el texto o leer la pregunta"), o como 'estrategias de lectura digital' (estrategias trabajadas en el entrenamiento).

Concretamente, la frecuencia de respuestas categorizadas como 'técnicas generales' o como 'estrategias de lectura digital', antes y después de la instrucción, varió de forma significativa, $\chi^{2}(3, N=20)=76,42, p<.01$. Los resultados reflejan un aumento en la frecuencia de estrategias de lectura digital mencionadas por los estudiantes antes (4) y después (78) de la intervención, lo que contrasta con las técnicas generales, que fueron mencionadas ampliamente antes de la instrucción (76), y menos tras la misma (36).

Un análisis detallado de las estrategias de lectura digital mencionadas tras la instrucción indica que los participantes en mayor medida mencionaban las estrategias de planificación y de revisión, y en menor medida las de evaluación y lectura profunda. La estrategia de activación de conocimientos previos, por su parte, no fue mencionada por ninguno de los alumnos.

Tabla 2. Frecuencia con la que las distintas técnicas y estrategias fueron mencionadas en el cuestionario de conocimientos declarativos, antes y después de la instrucción

$\begin{array}{lcc}\text { Técnicas generales } & \text { Pre-instrucción } & \text { Post-instrucción } \\ \text { Pasos tecnológicos } & 44 & 22 \\ \text { Cumplimiento de la tarea } & 32 & 15 \\ \text { Estrategias de lectura digital } & & \\ \text { Planificación } & 0 & 31 \\ \text { Evaluación } & 3 & 13 \\ \text { Revisión } & 0 & 22 \\ \text { Lectura profunda de informaciónrelevante } & 1 & 12 \\ \text { Activación de conocimientos previos } & 0 & 0\end{array}$




\section{Calidad de las Discusiones en la Tarea de Contrastar Casos}

Los resultados del análisis del contenido y grado de interacción de los estudiantes durante la discusión en pareja mostraron una correlación positiva entre el índice de calidad y el número de estrategias de lectura digital mencionas por los estudiantes tras la intervención, $r(18)=$ ,63, $p<, 01$. Al tratarse de una correlación, debemos ser cautos a la hora de establecer relaciones causales entre las variables. Por ejemplo, podría darse el caso que una tercera variable fuera la causante de una mejor discusión, y también de un buen aprendizaje. Para descartar una posible influencia en esta relación de lahabilidad lectora de los alumnos, medida con el PEL, correlacionamos esa medida con las dos variables de interés. No se encontraron correlaciones significativas ni con la calidad de las discusiones, $r(18)=, 18, p=, 43$, ni con el número de estrategias de lectura mencionadas tras la intervención, $r(18)=, 19, p<, 42$.

\section{Conclusiones}

Los resultados de este estudio exploratorio constatan que los alumnos de educación secundaria pueden aprender estrategias de lectura digital a partir de la instrucción con el video modelado de ejemplos a partir de movimientos oculares o EMMEs. Esta técnica se ha utilizado en un programa de forma que combinada otras técnicas instruccionales, por lo que podría considerarse como una técnica complementaria en los programas existentes en este ámbito (ArgelagósyPifarré, 2012; De Vries et al., 2008; Kuiper et al., 2008, 2009; Kuo y Hwang, 2008; Walraven et al., 2010).Por otro lado, esta técnica no solo aumenta las posibilidades de individualización de la tarea para cada estudiante, sino que también reduce la carga del docente (los videos se pueden consultar en cualquier momento, y se pueden ver en repetidas ocasiones sin que el tutor / modelo se canse).

Los alumnos que participaron en este programa instruccional aprendieron estrategias de lectura digital, en especial,la necesidad de planificar la búsqueda y la lectura, y revisar el plan de acción cuando sea necesario, seguidas por la lecturaen profundidad las páginas una vez identificada una sección con información relevante para la tarea y la evaluación de la relevancia de las secciones durante la búsqueda. Los estudiantes, sin embargo, no aprendieron la estrategia de activación de conocimientos previos. Posiblemente, el programa no enfatizó lo suficiente su importancia. Seguramente dicha estrategia debe trabajarse no como un aspecto aislado, sino como parte integrada de las otras estrategias.

En cualquier caso, los resultados mostraron que el programa instruccional con EMMEs no fue efectivo para todos los alumnos. La calidad de las discusiones por pareja estaba vinculada a un aprendizaje declarativo significativamente mejor de estrategias, aunque el efecto fue reducido. Esta relación es independiente de la habilidad lectora de los estudiantes, por lo que podemos descartar que se trate de un reflejo de la falta de competencia lectora de las parejas. Es posible que este efecto muestreel nivel de motivación de las parejas para llevar a cabo la actividad instruccional, lo que ya fue identificado por Bandura (1986) como uno de los condicionantes para la efectividad del aprendizaje por modelado.

\section{Limitaciones e Investigaciones Futuras}

Aunque los resultados sobre la técnica instruccional de los EMME son prometedores, nuestro estudio tiene un carácter exploratorio, por lo que sus resultados deben tratarse con cau- 
tela. En investigaciones futuras la efectividad de los EMME deberá analizarse en un estudio experimental que incluya un grupo control. Este grupo debería recibir instrucción mediante técnicas instruccionales utilizadas en nuestro programa (instrucción explícita, contraste de casos, aprendizaje por parejas y retroalimentación formativa), salvo los EMME. Asimismo, los resultados de nuestro estudio se limitan al aprendizaje declarativo de las estrategias, medido a partir de un cuestionario, por lo que no podemos concluir que la instrucción haya tenido un efecto en el aprendizaje procedimental de las mismas. En otras palabras, podemos afirmar que los alumnos conocen las estrategias, pero no que puedan ponerlas en práctica de forma eficaz. Para abordar este punto, futuras investigaciones deberían incluir la realización tareas de lectura digital antes y después de la intervención tanto de forma inmediata como demorada. Por último, y por lo que respecta a mejoras, con el fin de aumentar la motivación de loes estudiantes por la tarea, futuros estudios deberán utilizar materiales (textos digitales) con contenidos de mayor significatividad para los estudiantes de secundaria que los usados en el presente estudio.

Nuestro estudio abre la puerta al uso de los EMME para la instrucción de tareas cognitivas complejas, que impliquen no solo el trabajo con tareas visualmente complejas pero con instrucciones sencillas (e.g. Jarodzka et al., 2012, 2013), sino también el uso de estrategias de regulación durante la realización de las mismas.De esta forma, los EMMEs podrían ser de utilidad para amplios ámbitos de instrucción formal, tanto en entornos educativos como en el mundo de la empresa.

\section{Referencias}

Argelagós, E., yPifarré, M. (2012). Improving information problem solving skills in secondary education through embedded instruction.Computers in Human Behavior, 28(2), 515-526.

Bandura, A. (1986). Social foundations of thought and action: A social cognitive theory. Prentice-Hall, Inc.

Brand-Gruwel, S., Wopereis, I., yWalraven, A. (2009).A descriptive model of information problem solving while using internet.Computers\&Education, 53, 1207-1217.

Carrillo, M. y Marín, J. (1997). Prueba de Eficiencia Lectora.Documento interno sin publicar. Universidad de Murcia.

Coiro, J. (2011). Predicting reading comprehension on the internet contributions of offline reading skills, online reading skills, and prior knowledge. Journal of Literacy Research, 43(4), 352-392.

Coiro, J., y Dobler, E. (2007). Exploring the online reading comprehension strategies used by sixth-grade skilled readers to search for and locate information on the Internet. Reading ResearchQuarterly, 42(2), 214-257.

CONECTA (2014).Our Changing World - Lectura Digital.Estudio sobre los hábitos de lectura en dispositivos móviles realizado por CONECTA. http://www.conectarc.com/articulos-y-estudios/

De Vries, B., van der Meij, H.,yLazonder, A. W. (2008). Supporting reflective web searching in elementary schools. Computers in Human Behavior, 24(3), 649-665.

Eagleton, M. B., y Dobler, E. (2012). Reading the web: Strategies for Internet inquiry. New York: Guilford Press.

Jarodzka, H., Balslev, T., Holmqvist, K., Nyström, M., Scheiter, K., Gerjets, P., yEika, B. (2012). Conveying clinical reasoning based on visual observation via eye-movement modelling examples. Instructional Science, 40(5), 813-827. 
Jarodzka, H., van Gog, T., Dorr, M., Scheiter, K., y Gerjets, P. (2013). Learning to see: Guiding students' attention via a model's eye movements fosters learning. Learning and Instruction, 25, 62-70.

Kuiper, E., Volman, M., yTerwel, J. (2008). Students' use of Web literacy skills and strategies: searching, reading and evaluating Web information. Information research, 13(3), 5.

Kuiper, E., Volman, M., yTerwel, J. (2009).Developing Web literacy in collaborative inquiry activities.Computers \& Education, 52(3), 668-680.

Kuo, F. R., y Hwang, G. J. (2014). A five-phase learning cycle approach to improving the web-based problem-solving performance of students.Educational Technology \& Society, 17(1), 169-184.

Mason, L., Pluchino, P., y Tornatora, M. C. (2015). Using eye-tracking technologyas an indirect instruction tool to improve text and picture processing and learning.British Journal of Educational Technology, 41, 172-187.

Mason, L., Pluchino, P., y Tornatora, M. C. (2015). Eye-movement modeling of integrative reading of an illustrated text: Effects on processing and learning. Contemporary Educational Psychology, 41, 172-187.

Nicol, D. J., yMacfarlane-Dick, D. (2006). Formative assessment and self-regulated learning: A model and seven principles of good feedback practice. Studies in higher education, 31(2), 199-218.

Van Gog, T., Jarodzka, H., Scheiter, K., Gerjets, P., y Paas, F. (2009). Attention guidance during example study via the model's eye movements. Computers in Human Behavior, 25(3), 785-791.

Leu, D. J., Kinzer, C. K., Coiro, J. L.,y Cammack, D. W. (2004). Toward a theory of new literacies emerging from the Internet and other information and communication technologies.Theoretical models and processes of reading, 5, 1570-1613.

Nicol, D. J., y Macfarlane-Dick, D. (2006). Formative assessment and self-regulated learning: A model and seven principles of good feedback practice. Studies in HigherEducation, 31(2), 199-218.

OCDE (2013). PISA 2012. Programa para la evaluación internacional de los alumnos. Informe español. Volumen I: resultados y contexto. Madrid: Ministerio de educación, cultura y deporte.

Salmerón, L. (2013). Actividades que promueven la transferencia de los aprendizajes: una revisión de la literatura. Revista de Educación, volumen extraordinario 2013, 34-53.

Spatariu, A., Hartley, K., yBendixen, L. D. (2004). Defining and measuring quality in online discussions. The Journal of Interactive Online Learning, 2(4), 1-15.

Spires, H. A., Hervey, L. G., Morris, G., yStelpflug, C. (2012). Energizing project-based inquiry: middle grade students read, write, and create videos. Journal of Adolescent \& Adult Literacy, 55, 483-493.

Van Gog, T., y Rummel, N. (2010). Example-based learning: Integrating cognitive and social-cognitive research perspectives. Educational Psychology Review, 22(2), 155-174.

Walraven, A., Brand-Gruwel, S., yBoshuizen, H. P. (2010). Fostering transfer of websearchers' evaluation skills: A field test of two transfer theories. Computers in Human Behavior, 26(4), 716-728. 